\title{
A Comparative Study of Matrix Factorization and Random Walk with Restart in Recommender Systems
}

\author{
Haekyu Park \\ Computer Science and Engineering \\ Seoul National University \\ Seoul, Republic of Korea \\ Email: hkpark627@snu.ac.kr
}

\author{
Jinhong Jung \\ Computer Science and Engineering \\ Seoul National University \\ Seoul, Republic of Korea \\ Email: jinhongjung@ @nu.ac.kr
}

\author{
U Kang \\ Computer Science and Engineering \\ Seoul National University \\ Seoul, Republic of Korea \\ Email: ukang@snu.ac.kr
}

\begin{abstract}
Between matrix factorization or Random Walk with Restart (RWR), which method works better for recommender systems? Which method handles explicit or implicit feedback data better? Does additional information help recommendation? Recommender systems play an important role in many ecommerce services such as Amazon and Netflix to recommend new items to a user. Among various recommendation strategies, collaborative filtering has shown good performance by using rating patterns of users. Matrix factorization and random walk with restart are the most representative collaborative filtering methods. However, it is still unclear which method provides better recommendation performance despite their extensive utility.

In this paper, we provide a comparative study of matrix factorization and RWR in recommender systems. We exactly formulate each correspondence of the two methods according to various tasks in recommendation. Especially, we newly devise an RWR method using global bias term which corresponds to a matrix factorization method using biases. We describe details of the two methods in various aspects of recommendation quality such as how those methods handle cold-start problem which typically happens in collaborative filtering. We extensively perform experiments over real-world datasets to evaluate the performance of each method in terms of various measures. We observe that matrix factorization performs better with explicit feedback ratings while RWR is better with implicit ones. We also observe that exploiting global popularities of items is advantageous in the performance and that side information produces positive synergy with explicit feedback but gives negative effects with implicit one.
\end{abstract}

Index Terms-matrix factorization; random walk with restart; recommender systems

\section{INTRODUCTION}

Recommending new items to a user has been widely recognized as an important research topic in data mining area [1], [2], [3], [4], and recommender systems have been extensively applied in various applications across different domains to recommend items such as book [5], movie [6], [7], music [8], [9], friend [10], [11], scientific article [12], video [13], and restaurant [14]. Many e-commerce services such as Amazon and Netflix mainly depend on recommender systems to increase their profits by selling what consumers are interested in against overloaded information of products [15], [16], [7].

Collaborative filtering $(\mathrm{CF})$ has been successfully adopted among diverse recommendation strategies due to its high quality performance [17] and domain free property. For a query user, $\mathrm{CF}$ recommends items preferred by other users who present similar rating patterns to the query user [6]. This strategy does not require domain knowledge for recommendation, since it relies on only user history such as item ratings or previous transactions. However, the domain free property causes cold-start problem: the systems cannot recommend an item to a user if the item or the user are newly added to the system and existing data on them are not observed. Many techniques have been proposed [18], [19] to solve the coldstart problem, but the problem is still a major challenge in recommender systems.

The two major approaches of collaborative filtering are latent factor models and graph based models. Matrix factorization (MF) [6] is the most widely used method as a latent factor model, and it discovers latent factors inherent in relations between users and items by decomposing a user-item rating matrix. Random walk with restart (RWR) [20], [21], [22], [23], [24], [25] is commonly used as a graph based model for recommender systems [26], [11], [27]. RWR recommends items to a user with the user's personalized ordering of nodeto-node proximities which are measured by a random surfer in a user-item bipartite graph as shown in Figure 11. These methods also provide solutions for the cold start problem, using side information such as user demographic information or item category data [18], [19], [26], [28]. Although MF and RWR have been extensively used with the same purpose, it is still ambiguous to answer the following question: which method is better between matrix factorization and random walk with restart in recommender systems?

This paper aims to compare matrix factorization and random walk with restart in various tasks of recommendations with the corresponding metrics. We are interested in answering the following questions:

- Explicit feedback. Which method performs better when explicit feedback data are given?

- Implicit feedback. Which method performs better when implicit feedback data are given? 
- Bias terms. Do the bias terms improve the performance of recommendation methods? Which method performs better with bias factors?

- Side information. Does the side information improve the performance of recommendation methods? Which method is better when additional information are given?

In our experiments, matrix factorization performs better with explicit feedback data, while random walk with restart is better with implicit ones. We also observe that biases enhance the overall quality of recommendations. However, side information enhances the performance when used with explicit ratings, while degrades the performance with implicit ratings. Detailed explanations are stated in Section IV] The main contributions of this paper are as follows:

- Formulation. We show that each method in matrix factorization has its corresponding one in random walk with restart. This lays the groundwork for systematic comparison of the two methods.

- Method. We newly devise a random walk with restart method that introduces global bias terms. It reflects global popularities of items and general properties of users, improving random walk with restart methods without the bias terms.

- Analysis. We systemically compare the two collaborative filtering approaches. We also analyze properties of the methods that cause their strengths in various tasks.

- Experiments. We present and discuss extensive experimental results for many scenarios with various types of input over different recommendation purposes.

The code and datasets used in this paper are available at http://datalab.snu.ac.kr/mfrwr. The rest of this paper is organized as follows. Section II explains preliminaries on matrix factorization and random walk with restart for recommender systems. Section IIII presents recommendation methods that we discuss in this paper. Section IV shows experimental results and discussions on recommendation performance of the methods. Finally, we conclude this paper in Section $\mathrm{V}$

\section{PReliminaries}

Section $\Pi$ introduces preliminaries on matrix factorization and random walk with restart in recommender systems. Table II lists symbols and their definition used in this paper. We denote matrices and sets with upper-case bold letters (e.g. $\mathbf{R}$ or $\mathbf{U}$ ), vectors with lower-case bold letters (e.g. $\mathbf{x}_{u}$ ), scalars with lower-case italic letters (e.g. c), and graphs by upper-case normal letters (e.g. $G$ ).

\section{A. Matrix Factorization}

Matrix factorization (MF) predicts unobserved ratings given observed ratings. MF predicts a rating of item $i$ given by user $u$ as $\hat{r}_{u i}=\mathbf{x}_{u}^{T} \mathbf{y}_{i}$, where $\mathbf{x}_{u}$ is $u$ 's vector and $\mathbf{y}_{i}$ is $i$ 's vector.

Objective function is defined in Equation (1), where $r_{u i}$ is an observed rating and $\boldsymbol{\Omega}_{\mathbf{R}}$ is a set of (user, item) pairs for which ratings are observed. The term $\lambda\left(\left\|\mathbf{x}_{u}\right\|^{2}+\left\|\mathbf{y}_{i}\right\|^{2}\right)$ prevents overfitting by regularizing the magnitude of parameters, where the degree of regularization is controlled by the hyperparameter $\lambda$.
Table I: Table of symbols.

\begin{tabular}{cl}
\hline Symbol & Definition \\
\hline $\mathbf{U}$ & set of users \\
$\mathbf{I}$ & set of items \\
$\mathbf{R} \in \mathbb{R}^{|\mathbf{U}| \times|\mathbf{I}|}$ & observed rating matrix \\
$u$ & user \\
$i$ & item \\
$r_{u i}$ & observed rating of $u$ on $i$ \\
$\hat{r}_{u i}$ & predicted rating of $u$ on $i$ \\
$\alpha$ & coefficient of confidence level in implicit feedback \\
$\mu$ & average of ratings \\
$b_{u}$ & bias of $u$ \\
$b_{i}$ & bias of $i$ \\
$s$ & a user attribute \\
$a_{u s}$ & user attribute of $u$ with respect to $s$ \\
$t$ & an item attribute \\
$b_{t i}$ & item attribute of $i$ with respect to $t$ \\
$\boldsymbol{\Omega}_{\mathbf{R}}$ & set of $(u, i)$ where $r_{u i}$ is observed \\
$\boldsymbol{\Omega}_{\mathbf{A}}$ & set of $(u, s)$ where $a_{u s}$ is observed \\
$\boldsymbol{\Omega}_{\mathbf{B}}$ & set of $(t, i)$ where $b_{t i}$ is observed \\
$d$ & dimension of latent vectors of users and items \\
$\mathbf{x}_{u} \in \mathbb{R}^{d}$ & vector of user $u$ \\
$\mathbf{y}_{i} \in \mathbb{R}^{d}$ & vector of item $i$ \\
$\mathbf{w}_{s} \in \mathbb{R}^{d}$ & vector of user attribute $s$ \\
$\mathbf{z}_{t} \in \mathbb{R}^{d}$ & vector of item attribute $t$ \\
$\lambda$ & regularization parameter \\
$\eta$ & learning rate \\
$G=(\mathbf{V}, \mathbf{E})$ & user-item bipartite graph \\
$\mathbf{V}$ & set of nodes in $G$, i.e., $\mathbf{V}=\mathbf{U} \cup \mathbf{I}$ \\
$\mathbf{E}$ & set of weighted edges $\left(u, i, r_{u i}\right)$ in $G$ \\
$\mathbf{A}$ & adjacency matrix of $G$ \\
$\tilde{\mathbf{A}}$ & row-normalized adjacency matrix of $\mathbf{A}$ \\
$G^{\prime}$ & augmented graph adding side info. into $G$ \\
$\delta$ & weight of link representing side info. in $G^{\prime}$ \\
$c$ & restart probability in RWR \\
$\mathbf{q}$ & starting vector in RWR \\
$\mathbf{b}$ & bias vector in RWR \\
$\beta$ & walk coefficient in biased RWR \\
$\gamma$ & jump coefficient in biased RWR \\
\hline & \\
\hline & \\
\hline &
\end{tabular}

$L=\frac{1}{2} \sum_{(u, i) \in \mathbf{\Omega}_{\mathbf{R}}}\left(\left(r_{u i}-\mathbf{x}_{u}^{T} \mathbf{y}_{i}\right)^{2}+\lambda\left(\left\|\mathbf{x}_{u}\right\|^{2}+\left\|\mathbf{y}_{i}\right\|^{2}\right)\right)$

The standard approach to learn parameters which minimize $L$ is GD (Gradient Descent). The update procedures for parameters in GD are as follows.

$$
\begin{array}{cl}
\mathbf{x}_{u} \leftarrow \mathbf{x}_{u}-\eta \nabla_{\mathbf{x}_{u}} L, & \nabla_{\mathbf{x}_{u}} L=-e_{u i} \mathbf{y}_{i}+\lambda \mathbf{x}_{u} \\
\mathbf{y}_{i} \leftarrow \mathbf{y}_{i}-\eta \nabla_{\mathbf{y}_{i}} L, & \nabla_{\mathbf{y}_{i}} L=-e_{u i} \mathbf{x}_{u}+\lambda \mathbf{y}_{i}
\end{array}
$$

, where $\eta$ is a learning rate and $e_{u i}$ is a prediction error defined as $e_{u i}=r_{u i}-\mathbf{x}_{u}^{T} \mathbf{y}_{i}$.

\section{B. Random Walk with Restart}

Random Walk with Restart (RWR) is one of the most commonly used methods for graph based collaborative filtering in recommender systems [26], [28]. Given a user-item bipartite graph $G$ and a query user $u$ as seen in Figure 11. RWR computes a personalized ranking of items w.r.t the user $u$. The input graph $G$ comprises the set of nodes $\mathbf{V}$ with the set of users $\mathbf{U}$ and the set of items $\mathbf{I}$, i.e., $\mathbf{V}=\mathbf{U} \cup \mathbf{I}$. Each edge $\left(u, i, r_{u i}\right) \in \mathbf{E}$ represents the rating $r_{u i}$ between user $u$ and item $i$, and the rating is the weight of the edge.

RWR exploits a random surfer to produce the personalized ranking of items for a user $u$ by letting her move around the 


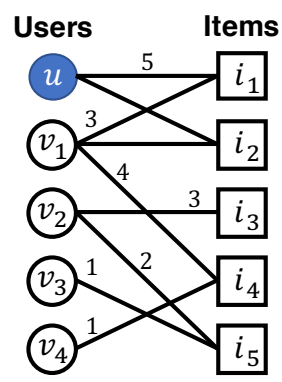

Fig. 1: Example of a user-item bipartite graph. In the graph, edges between users and items are weighted with ratings. Node $u$ is a starting node for RWR which measures node-to-node proximities w.r.t. node $u$ to rank items.

Table II: Correspondence between matrix factorization (MF) and random walk with restart (RWR) according to tasks.

\begin{tabular}{llc}
\hline Task & MF & RWR \\
\hline Explicit feedback & $\mathrm{MF}_{\text {Exp }}$ (Section III-A1) & $\mathrm{RWR}_{\text {Exp }}$ (Section III-B1) \\
Implicit feedback & $\mathrm{MF}_{\text {Imp }}$ (Section III-A2) & $\mathrm{RWR}_{\text {Imp }}$ (Section III-B2) \\
Global bias & $\mathrm{MF}_{\text {Bias }}$ (Section III-A3 & $\mathrm{RWR}_{\text {Bias }}$ (Section III-B3) \\
Using side info. & $\mathrm{MF}_{\text {Side }}$ (Section III-A44 & $\mathrm{RWR}_{\text {Side }}$ (Section III-B4) \\
\hline
\end{tabular}

graph $G$. Suppose the random surfer started from the user node $u$ and she is at the node $v$ currently. Then the surfer takes one of the following actions: random walk or restart. Random walk indicates that the surfer moves to a neighbor node of the current node with probability $1-c$, and restart indicates that the surfer goes back to the starting node $u$ with probability $c$. If the random surfer visits node $v$ many times, then node $v$ is highly related to node $u$; thus, node $v$ is ranked high in the personalized ranking for $u$.

The random surfer is likely to frequently visit items that are rated highly by users who give ratings similarly to the query user $u$, which is consistent with the intuition of collaborative filtering, i.e., if a user has similar taste with the query user $u$, and the similar user likes an item $i$, then $u$ is likely prefer the item $i$. We measure probabilities that the random surfer visits each item as ranking scores, called RWR scores, and sort the scores in the descending order to recommend items for the query user $u$. The detailed method for computing RWR is described in Section $\amalg I I-\mathrm{B}$.

\section{RECOMMENDATION METHODS}

Section III describes recommendation methods to be compared in this paper. They are based on matrix factorization (Section II-A) or random walk with restart (Section II-B). We present four methods for each approach in cases of the following scenario list. We suggest a matrix factorization method and a random walk with restart method for each scenario, and they are summarized in Table

- Explicit feedback. We recommend items when explicit feedback ratings are given; for example, movie rating data with 1 to 5 scaled "stars" are given.

- Implicit feedback. We also present recommendation methods for implicit feedback data such as the number of clicks on items.

- Global bias terms. Bias terms indicating global properties of users and items are used in recommendation methods to predict preferences of users more accurately.

- Employing side information. We present recommendation methods that use auxiliary information. Auxiliary information of users and items is used to solve cold start problem or enhance accuracy of recommendations.

\section{A. Recommendation Methods Based on Matrix Factorization}

We explain four methods based on matrix factorization as follows. Each of them is different in its purpose and type of datasets they use.

\section{1) $\mathrm{MF}_{\text {Exp }}$, A Basic Matrix Factorization Method for Explicit Rating}

$\mathrm{MF}_{\text {Exp }}$ is a standard matrix factorization based method for explicit feedback ratings [6]. It predicts unobserved ratings by learning embeddings of users and items. $\mathrm{MF}_{\mathrm{Exp}}$ is described in section $\amalg-\mathrm{A}$.

\section{2) $\mathrm{MF}_{\mathrm{Imp}}$, A Basic Matrix Factorization Method for Implicit} Rating

$\mathrm{MF}_{\text {Imp }}$ is for implicit feedback ratings [29]. For a user $u$, an item $i$, and an implicit feedback rating $r_{u i}, \mathrm{MF}_{\text {Imp }}$ predicts a binarized implicit feedback rating $p_{u i}$ by learning $u$ 's vector $\mathbf{x}_{u}$ and $i$ 's vector $\mathbf{y}_{i}$. The implicit feedback rating $r_{u i}$ is the number of times that a user performs a favorable action to an item, e.g. the number of clicks of the item. The binarized implicit feedback rating $p_{u i}$ is defined as 1 if $r_{u i}>0$ and 0 otherwise.

$\mathrm{MF}_{\text {Imp }}$ requires confidence levels of each rating $r_{u i}$, since implicit feedback data are inherently noisy. For example, not watching a movie occasionally indicates dislike for the movie or ignorance of its existence. The confidence level of a rating crystallizes how certainly we can use the rating value. We define the confidence level of an implicit rating for $u$ and $i$ as $c_{u i}=1+\alpha r_{u i}$ as stated in [29].

The objective function of $\mathrm{MF}_{\mathrm{Imp}}$ is defined in Equation (2). $c_{u i}$ adjusts intensity of learning $\mathbf{x}_{u}$ and $\mathbf{y}_{i}$ in gradient descent update of the vectors as presented in Equations (3) and (4), where $e_{u i}$ is a prediction error defined as $e_{u i}=p_{u i}-\mathbf{x}_{u}^{T} \mathbf{y}_{i}$.

$$
\begin{gathered}
L=\frac{1}{2} \sum_{(u, i) \in \mathbf{\Omega}_{\mathbf{R}}}\left(c_{u i}\left(p_{u i}-\mathbf{x}_{u}^{T} \mathbf{y}_{i}\right)^{2}+\lambda\left(\left\|\mathbf{x}_{u}\right\|^{2}+\left\|\mathbf{y}_{i}\right\|^{2}\right)\right) \\
\mathbf{x}_{u} \leftarrow \mathbf{x}_{u}-\eta \nabla_{\mathbf{x}_{u}} L, \quad \nabla_{\mathbf{x}_{u}} L=-e_{u i} c_{u i} \mathbf{y}_{i}+\lambda \mathbf{x}_{u} \\
\mathbf{y}_{i} \leftarrow \mathbf{y}_{i}-\eta \nabla_{\mathbf{y}_{i}} L, \quad \nabla_{\mathbf{y}_{i}} L=-e_{u i} c_{u i} \mathbf{x}_{u}+\lambda \mathbf{y}_{i}
\end{gathered}
$$

3) $\mathrm{MF}_{\text {Bias }}$, A Matrix Factorization Method with Global Bias Terms

$\mathrm{MF}_{\text {Bias }}$ introduces bias terms into $\mathrm{MF}_{\text {Exp }}$ to represent individual rating pattern of users and items. For example, a user $u$ 's bias term $b_{u}$ is learned to possess a high value if $u$ normally rates all items favorably. An item $i$ 's bias $b_{i}$ is learned to have a high value if it is rated highly by almost all users.

The objective function of $\mathrm{MF}_{\mathrm{Bias}}$ is given in Equation (5). $\mu$ is a global average rating value and $b_{e}$ is a bias term of a user or an item $e$. 


$$
\begin{aligned}
L=\frac{1}{2} \sum_{(u, i) \in \boldsymbol{\Omega}_{\mathbf{R}}} & \left(\left(r_{u i}-\mu-b_{u}-b_{i}-\mathbf{x}_{u}^{T} \mathbf{y}_{i}\right)^{2}\right. \\
& \left.+\lambda\left(b_{u}^{2}+b_{i}^{2}+\left\|\mathbf{x}_{u}\right\|^{2}+\left\|\mathbf{y}_{i}\right\|^{2}\right)\right)
\end{aligned}
$$

We use GD (Gradient Descent) method to minimize $L$ in Equation (5). The update procedures for parameters are as follows.

$$
\begin{array}{cc}
b_{u} \leftarrow b_{u}-\eta \frac{\partial L}{\partial b_{u}}, & \frac{\partial L}{\partial b_{u}}=-e_{u i}+\lambda b_{u} \\
b_{i} \leftarrow b_{i}-\eta \frac{\partial L}{\partial b_{i}}, & \frac{\partial L}{\partial b_{i}}=-e_{u i}+\lambda b_{i} \\
\mathbf{x}_{u} \leftarrow \mathbf{x}_{u}-\eta \nabla_{\mathbf{x}_{u}} L, & \nabla_{\mathbf{x}_{u}} L=-e_{u i} \mathbf{y}_{i}+\lambda \mathbf{x}_{u} \\
\mathbf{y}_{i} \leftarrow \mathbf{y}_{i}-\eta \nabla_{\mathbf{y}_{i}} L, & \nabla_{\mathbf{y}_{i}} L=-e_{u i} \mathbf{x}_{u}+\lambda \mathbf{y}_{i}
\end{array}
$$

, where $e_{u i}$ is a prediction error defined as $e_{u i}=r_{u i}-\mu-$ $b_{u}-b_{i}-\mathbf{x}_{u}^{T} \mathbf{y}_{i}$.

4) $\mathrm{MF}_{\text {Side, }}$ A Coupled Matrix Factorization Method Using Side Information

$\mathrm{MF}_{\text {Side }}$ uses additional information to understand users and items in many-sided properties. User similarity information such as friendship in a social network or item similarity information such as items' category is advantageous for finding users with similar tastes and items with similar properties, which causes latent vectors of users and items to be similar. Side information is useful when rating data of users and items are missing. For example, if a cold-start user $u$ has similar demographic information with another warm-user $v, \mathbf{x}_{u}$ is able to be learned to be similar to $\mathbf{x}_{v}$ even though $u$ has never rated an item.

The objective function of $\mathrm{MF}_{\text {Side }}$ is defined in Equation (6).

$$
\begin{aligned}
L & =\overbrace{\frac{1}{2} \sum_{(u, i) \in \boldsymbol{\Omega}_{\mathbf{R}}}\left(\left(r_{u i}-\mathbf{x}_{u}^{T} \mathbf{y}_{i}\right)^{2}+\lambda\left(\left\|\mathbf{x}_{u}\right\|^{2}+\left\|\mathbf{y}_{i}\right\|^{2}\right)\right)}^{\text {(1) Factorization of ratings }} \\
+ & \overbrace{\frac{1}{2} \sum_{(u, s) \in \boldsymbol{\Omega}_{\mathbf{A}}}\left(\left(a_{u s}-\mathbf{x}_{u}^{T} \mathbf{w}_{s}\right)^{2}+\lambda\left(\left\|\mathbf{x}_{u}\right\|^{2}+\left\|\mathbf{w}_{s}\right\|^{2}\right)\right)}^{\text {Factorization of user similarity attributes }}
\end{aligned}
$$

(3) Factorization of item similarity attributes

$$
+\overbrace{\frac{1}{2} \sum_{(t, i) \in \boldsymbol{\Omega}_{\mathrm{B}}}\left(\left(b_{t i}-\mathbf{z}_{t}^{T} \mathbf{y}_{i}\right)^{2}+\lambda\left(\left\|\mathbf{z}_{t}\right\|^{2}+\left\|\mathbf{y}_{i}\right\|^{2}\right)\right)}
$$

$\boldsymbol{\Omega}_{\mathbf{R}}$ is a set of (user, item) where ratings are observed. $\boldsymbol{\Omega}_{\mathbf{A}}$ is a set of (user, user attribute) where the user similarity attributes are observed. $\Omega_{\mathrm{B}}$ is a set of (item attribute, item) where the item similarity attributes are observed. For a user $u$ and a user similarity attribute $s, a_{u s}$ indicates $u$ 's properties on $s$ such as $u$ 's age. $\mathbf{w}_{s}$ is a latent vector of $s . \mathbf{x}_{u}$ is $u$ 's latent feature that couples ratings and user attributes. For an item $i$ and an item similarity attribute $t, b_{t i}$ is $i$ 's attribute value with respect to $t$ such as $i$ 's category if $t$ indicates category of items. $\mathbf{z}_{t}$ is a latent vector of $t$, and $\mathbf{y}_{i}$ is a latent vector of $i$ that represents ratings and item similarity attributes of $i$ simultaneously.

We use the following gradient descent updates for $\mathbf{x}_{u}, \mathbf{y}_{i}$, $\mathbf{w}_{s}$, and $\mathbf{z}_{t}$. For a user $u$ and an item $i$ for $(u, i) \in \boldsymbol{\Omega}_{\mathbf{R}}$, we update $\mathbf{x}_{u}$ and $\mathbf{y}_{i}$ as follows, where $e_{u i}=r_{u i}-\mathbf{x}_{u}^{T} \mathbf{y}_{i}$.

$$
\begin{array}{cc}
\mathbf{x}_{u} \leftarrow \mathbf{x}_{u}-\eta \nabla_{\mathbf{x}_{u}}(1), \quad \nabla_{\mathbf{x}_{u}}(1)=-e_{u i} \mathbf{y}_{i}+\lambda \mathbf{x}_{u} \\
\mathbf{y}_{i} \leftarrow \mathbf{y}_{i}-\eta \nabla_{\mathbf{y}_{i}} \text { (1), } \quad \nabla_{\mathbf{y}_{i}}(1)=-e_{u i} \mathbf{x}_{u}+\lambda \mathbf{y}_{i}
\end{array}
$$

For a user $u$ and a user similarity attribute $s$ for $(u, s) \in \boldsymbol{\Omega}_{\mathbf{A}}$, we update $\mathbf{x}_{u}$ and $\mathbf{w}_{s}$ as follows, where $e_{u s}=a_{u s}-\mathbf{x}_{u}^{T} \mathbf{w}_{s}$.

$$
\begin{array}{ll}
\mathbf{x}_{u} \leftarrow \mathbf{x}_{u}-\eta \nabla_{\mathbf{x}_{u}}(2), & \nabla_{\mathbf{x}_{u}}(2)=-e_{u s} \mathbf{w}_{s}+\lambda \mathbf{x}_{u} \\
\mathbf{w}_{s} \leftarrow \mathbf{w}_{s}-\eta \nabla_{\mathbf{w}_{s}}(2), & \nabla_{\mathbf{w}_{s}}(2)=-e_{u s} \mathbf{x}_{u}+\lambda \mathbf{w}_{s}
\end{array}
$$

For an item similarity attribute $t$ and an item $i$ for $(t, i) \in \boldsymbol{\Omega}_{\mathbf{B}}$, we update $\mathbf{z}_{t}$ and $\mathbf{y}_{i}$ as follows, where $e_{t i}=b_{t i}-\mathbf{z}_{t}^{T} \mathbf{y}_{i}$.

$$
\begin{array}{ll}
\mathbf{z}_{t} \leftarrow \mathbf{z}_{t}-\eta \nabla_{\mathbf{z}_{t}} \text { (3), } & \nabla_{\mathbf{z}_{t}} \text { (3) }=-e_{t i} \mathbf{y}_{i}+\lambda \mathbf{z}_{t} \\
\mathbf{y}_{i} \leftarrow \mathbf{y}_{i}-\eta \nabla_{\mathbf{y}_{i}} \text { (3), } & \nabla_{\mathbf{y}_{i}} \text { (3) }=-e_{t i} \mathbf{z}_{t}+\lambda \mathbf{y}_{i}
\end{array}
$$

\section{B. Recommendation Methods Based on Random Walk with Restart}

We explain four methods based on random walk with restart according to rating types of input data and their purposes.

1) $\mathrm{RWR}_{\mathrm{Exp}}$, A Basic Random Walk with Restart Method for Explicit Rating

Suppose we have a user-item bipartite graph $G=(\mathbf{V}, \mathbf{E})$ where $\mathbf{V}$ is the set of nodes, and $\mathbf{E}$ is the set of edges. $\mathbf{V}$ consists of users and items, i.e., $\mathbf{V}=\mathbf{U} \cup \mathbf{I}$ where $\mathbf{U}$ is the set of users and $\mathbf{I}$ is the set of items. Each edge $\left(u, i, r_{u i}\right) \in \mathbf{E}$ represents a rating between user $u$ and item $i$, and the edge is weighted with the rating $r_{u i}$. For a starting node $u$, the RWR scores are defined as the following recursive equation [20]:

$$
\mathbf{r}=(1-c) \tilde{\mathbf{A}}^{\top} \mathbf{r}+c \mathbf{q}
$$

where $\mathbf{r} \in \mathbb{R}^{|\mathbf{V}|}$ is the RWR score vector w.r.t. the starting node $u, \mathbf{q} \in \mathbb{R}^{|\mathbf{V}|}$ is the starting vector whose $u$-th entry is 1 and all other entries are 0 , and $c$ is the restarting probability. $\tilde{\mathbf{A}}$ is the row-normalized adjacency matrix, i.e., $\tilde{\mathbf{A}}=\mathbf{D}^{-1} \mathbf{A}$ where $\mathbf{A}$ is the weighted adjacency matrix of the graph $G$, and $\mathbf{D}$ is a diagonal matrix of weighted degrees such that $\mathbf{D}_{i i}=\sum_{j} \mathbf{A}_{i j}$ and $\mathbf{A}_{i j}=r_{i j}$ indicating the weighted edge $\left(i, j, r_{i j}\right) \in \mathbf{E}$.

The RWR score vector $\mathbf{r}$ is iteratively updated as follows:

$$
\mathbf{r}^{(t)} \leftarrow(1-c) \tilde{\mathbf{A}}^{\top} \mathbf{r}^{(t)}+c \mathbf{q}
$$

where $\mathbf{r}^{(t)}$ is the RWR score vector of $t$-th iteration. The iteration starts with the initial RWR score vector $\mathbf{r}^{(0)}$, and it is repeated until convergence (i.e., the iteration stops when $\left\|\mathbf{r}^{(t)}-\mathbf{r}^{(t-1)}\right\|<\epsilon$ where $\epsilon$ is the error tolerance). The iteration for $\mathbf{r}^{(t)}$ converges to a unique solution [30]. We initialize $\mathbf{r}^{(0)}$ as $\frac{1}{|\mathbf{V}|} \mathbf{1}$ where $|\mathbf{V}|$ is the number of nodes and $\mathbf{1} \in \mathbb{R}^{|\mathbf{V}|}$ is an all-ones vector. Note that for each user, we compute the RWR score vector $\mathbf{r}$, and rank items in the order of the RWR scores on items $\mathbf{r}_{i}$ s.t. $i \in \mathbf{I}$. 


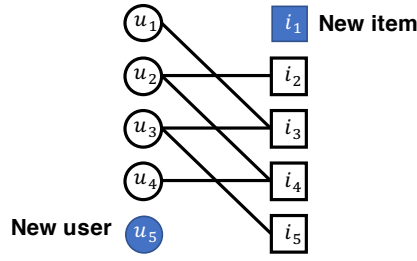

(a) Cold-start problem

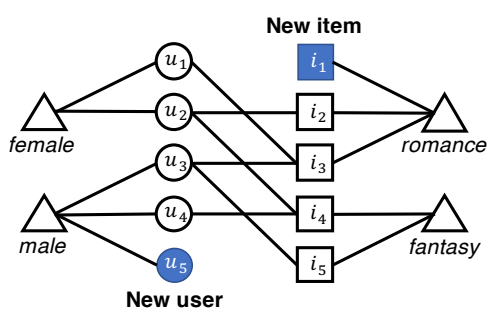

(b) Augmented graph using side information

Fig. 2: Examples of the cold-start problem in a user-item bipartite graph (a) and the augmented bipartite graph (b). In (a), user $u_{5}$ and item $i_{1}$ have the cold-start problem since they do not have edges with other users or items. (b) shows how the augmented graph resolves the cold-start problem using side information such as user demographic information or item category data.

\section{2) $\mathrm{RWR}_{\mathrm{Imp}}$, A Basic Random Walk with Restart Method for} Implicit Rating

When explicit user-item ratings are not available, RWR is also able to exploit implicit feedback ratings described in Section III-A2 for recommendation. Instead of constructing the user-item bipartite graph using the explicit ratings, we build the graph $G$ based on the implicit feedback ratings. An edge between user $u$ and item $i$ in the graph $G$ is represented as $\left(u, i, c_{u i}\right)$ indicating an implicit rating for user $u$ and item $i$. $c_{u i}$ is the confidence level of the implicit rating as explained in Section III-A2 Then we compute RWR scores in the user-item bipartite graph $G$ using Equation (8).

3) $\mathrm{RWR}_{\text {Bias }}$, A Random Walk with Restart Method with Global Bias Terms

As described in Section III-B1, the traditional RWR methods do not consider global user and item popularity even if the popularity affects the performance of recommendation as discussed in [6]. Thus we propose a novel random walk with restart method RWR $\mathrm{Bias}_{\text {for }}$ fonsidering the global popularity (or bias) for users and items as well as personalized information in recommendation.

For a query user $u$, the goals of $\mathrm{RWR}_{\text {Bias }}$ are stated as follows: (1) it obtains RWR scores of other nodes with respect to $u$ which indicate similarity with $u$, and (2) it considers global popularities of users and items in RWR scores. RWR Bias builds on top of a basic RWR method by adding bias terms to achieve the above goals. The first goal of calculating similarity score is done by the basic RWR approach. Bias terms are implemented to achieve the second goal to increase RWR scores of generally popular items or users while decreasing the scores of generally unpopular ones.

The iterative equations to update RWR score vector and bias vectors are defined in Equations (11) and (9), respectively. The $\mathrm{RWR}_{\text {Bias }}$ score vector $\mathbf{r}$ consists of RWR terms as $\beta \tilde{\mathbf{A}}^{\top} \mathbf{r}+\gamma \mathbf{q}$ and bias terms $(1-\beta-\gamma) \mathbf{b}$. The bias vector $\mathbf{b} \in \mathbb{R}^{|\mathbf{V}|}$ consists of the random walk term as $(1-c) \tilde{\mathbf{A}}^{\top} \mathbf{b}$ and a jump term as $c \tilde{\mathbf{m}}$ where $\tilde{\mathbf{m}}$ is aimed to increase RWR scores of popular nodes by assigning higher probabilities that a random surfer jumps to the popular entities. $\tilde{\mathbf{m}}$ is normalized to sum to 1 from a non-normalized vector $\mathbf{m}$; for each entity $e, e$-th entry value in $\mathbf{m}$ is the global average rating value of the corresponding type of entity $e$, i.e., the average of the ratings that an item receives or the average of the ratings that a user gives.

We first compute the bias vector $\mathbf{b}$ as follows:

$$
\mathbf{b}=(1-c) \tilde{\mathbf{A}}^{\top} \mathbf{b}+c \tilde{\mathbf{m}} \Leftrightarrow \mathbf{b}=\mathbf{G}^{\prime} \mathbf{b}
$$

where $\mathbf{G}^{\prime}=(1-c) \tilde{\mathbf{A}}^{\top}+c \tilde{\mathbf{m}} \mathbf{1}^{\top}$ and $\mathbf{1}$ is an all-ones column vector. Note that $\mathbf{G}^{\prime}$ is a column stochastic matrix if $\tilde{\mathbf{A}}^{\top}$ is column stochastic, since $\mathbf{1}^{\top} \mathbf{G}^{\prime}=\mathbf{1}^{\top}$. Also the sum of each entry of $\mathbf{b}$ is 1 (i.e., $\mathbf{1}^{\top} \mathbf{b}=1$ ), which is proved as follows:

$$
\begin{aligned}
& \mathbf{1}^{\top} \mathbf{b}=(1-c) \mathbf{1}^{\top} \tilde{\mathbf{A}}^{\top} \mathbf{b}+c \mathbf{1}^{\top} \tilde{\mathbf{m}}=(1-c) \mathbf{1}^{\top} \mathbf{b}+c \\
& \Leftrightarrow c \mathbf{1}^{\top} \mathbf{b}=c \Leftrightarrow \mathbf{1}^{\top} \mathbf{b}=1
\end{aligned}
$$

Then, we compute the RWR Bias score vector $\mathbf{r}$ as follows:

$$
\mathbf{r}=\beta \tilde{\mathbf{A}}^{\top} \mathbf{r}+\gamma \mathbf{q}+(1-\beta-\gamma) \mathbf{b} \Leftrightarrow \mathbf{r}=\mathbf{G r}
$$

where $\mathbf{G}=\beta \tilde{\mathbf{A}}^{\top}+\gamma \mathbf{q} \mathbf{1}^{\top}+(1-\beta-\gamma) \mathbf{b} \mathbf{1}^{\top}$. Note that $\mathbf{G}$ is also column stochastic if $\tilde{\mathbf{A}}^{\top}$ is a column stochastic matrix since $\mathbf{1}^{\top} \mathbf{G}=\mathbf{1}^{\top}$, and $\mathbf{1}^{\top} \mathbf{r}=1$ which is proved similarly to Equation (10).

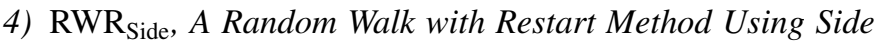
Information

The basic RWR described in Section [II-B1 also suffers from the cold-start problem since there are no out-going edges from a new user or a new item as shown in Figure 2(a) In this case, a random surfer is stuck in the new user node or the surfer cannot reach at the new item node; thus, the basic RWR cannot compute a personalized ranking of items for the new user, and the newly added item is omitted from recommendation list.

The main idea to solve the cold-start problem is to augment the user-item bipartite graph $G$ using side information such as user demographic information or item category data [26], [28]. Figure 2(b) depicts the example of how to augment the graph using side information. Suppose we have gender information of users. Then we add female and male nodes into the graph $G$, and connect gender nodes and users nodes as in Figure 2(b) Item nodes are also augmented similarly, using item information such as item category data.

Let $G^{\prime}$ denote the augmented graph. We add additional information into $G^{\prime}$ as links with weights set to $\delta$. Then RWR $_{\text {Side }}$ computes RWR scores on the augmented graph $G^{\prime}$ based on Equation (7). Note that in Equation (7), we normalize the adjacency matrix of the augmented graph $G^{\prime}$. RWR Side $_{\text {also }}$ ranks items in the order of the RWR scores on items $\mathbf{r}_{i}$ s.t. $i \in \mathbf{I}$ to generate an item recommendation list for each user.

\section{EXPERIMENTS}

We conduct extensive experiments to compare recommendation methods which are based on matrix factorization or 
random walk with restart. We focus on the following research questions according to the major tasks and present answers we got from the experiments.

- Explicit feedback (Section IV-C)

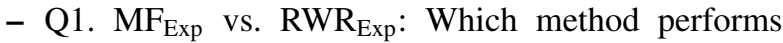
better when using explicit feedback data?

* A1. $\mathrm{MF}_{\text {Exp }}$ is better.

- Implicit feedback (Section IV-D

- Q2. $\mathrm{MF}_{\text {Imp }}$ vs. RWR Imp: Which method performs better when using implicit feedback data?

* A2. $\mathrm{RWR}_{\text {Imp }}$ is better.

- Global bias terms (Section IV-E

- Q3. Do global bias terms improve performance? * A3. Yes.

- Q4. $\mathrm{MF}_{\text {Bias }}$ vs. RWR $\mathrm{Bias}_{\text {: Which method performs }}$ better when exploiting global bias terms?

* A4. MF $\mathrm{Bias}_{\text {is }}$ better.

- Employing side information (Section IV-F)

- Q5. Does side information enhance performance?

* A5. Yes with explicit ratings, and No with implicit ratings.

- Q6. $\mathrm{MF}_{\text {Side }}$ vs. RWR $\mathrm{R}_{\text {Side }}$ : Which method performs better when employing side information?

* A6. MF Side $_{\text {is better with explicit rating data and }}$ $\mathrm{RWR}_{\text {Side }}$ is better with implicit rating data.

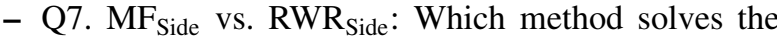
cold start problem better when employing side data?

* A7. $\mathrm{MF}_{\text {Side }}$ is better in explicit rating data and $\mathrm{RWR}_{\text {Side }}$ is better in implicit rating data.

A. Experimental Setup

1) Datasets

We use various rating datasets such as explicit rating data, implicit rating data, and ratings with additional information. We summarize the datasets in Tables III and IV]

- Movielens (https://movielens.org) is a movie recommendation site. It contains $0.9 \mathrm{~K}$ users and $1.6 \mathrm{~K}$ items. Ratings are in a range from 1.0 to 5.0, and their unit interval is 1.0. It provides user demographic information such as age, gender, occupation, and zip code. The dataset is available at https://grouplens.org/datasets/movielens/100k/.

- FilmTrust is a movie sharing and rating website. This dataset is crawled by Guo et al. [31]. It contains $1.6 \mathrm{~K}$ users and $2 \mathrm{~K}$ items where users are connected by directed edges in a trust network. Ratings are in a range from 0.5 to 4.0 , and their unit interval is 0.5 . The dataset is available at https://www.librec.net/datasets.html

- Epinions (epinions.com) is a who-trust-whom online social network of general consumer reviews. It contains $49 \mathrm{~K}$ users and $140 \mathrm{~K}$ items where users are connected by directed edges in a trust network. Ratings are in a range from 1.0 to 5.0, and their unit interval is 1.0. This dataset is available at http://www.trustlet.org/epinions.html

- Lastfm (https://www.last.fm) is an online music site for free music data such as music, video, photos, concerts, and so on. It includes $1.9 \mathrm{~K}$ users, $17.6 \mathrm{~K}$ items, $92.8 \mathrm{~K}$
Table III: Statistics of datasets.

\begin{tabular}{lrrrc}
\hline & \# of users & \# of items & \# of ratings & rating types \\
\hline Movielens & 943 & 1,682 & 100,000 & explicit \\
FilmTrust & 1,642 & 2,071 & 35,494 & explicit \\
Epinions & 49,289 & 139,738 & 664,824 & explicit \\
\hline Lastfm & 1,892 & 17,632 & 92,834 & implicit \\
Audioscrobbler & 148,111 & $1,631,028$ & $24,296,858$ & implicit \\
\hline
\end{tabular}

Table IV: Description of side information of datasets.

\begin{tabular}{lll}
\hline & types of side info & details \\
\hline Movielens & user demographic & age, gender, occupation, and zip code \\
FilmTrust & information & of all users \\
Epinions & social network & 1,309 social links \\
Lastfm & social network & 664,824 social links \\
Audioscrobbler & social network & N/A \\
\hline
\end{tabular}

implicit ratings, and $25.4 \mathrm{~K}$ social links. Items are artists in this dataset, and ratings are the number of times that a user listens to the artists' music. The dataset is available at https://grouplens.org/datasets/hetrec-2011/.

- Audioscrobbler (http://www.audioscrobbler.net) is a database system that tracks people's habits of listening to music. The dataset contains $148 \mathrm{~K}$ users, $1,631 \mathrm{~K}$ items, $24,297 \mathrm{~K}$ implicit ratings. Side information is not provided. The dataset is available at http://www-etud. iro.umontreal.ca/ bergstrj/audioscrobbler_data.html

\section{2) Cross-validation}

We separate training and test sets under 5-folded cross validation for all experiments except other ones related to the cold start problem; the detailed setting for the cold start problem is in Section IV-F. It ensures that each observed rating is included in a test set only once.

\section{3) Hyperparameters}

We find out hyperparameters which give the best performance for each experiment. They are set as follows. We use $\alpha=0.0001$ for Lastfm and Audioscrobbler in all case, and $d=5$ for all matrix factorization methods. In $\mathrm{MF}_{\mathrm{Exp}}, \mathrm{MF}_{\mathrm{Imp}}$, and $\mathrm{MF}_{\text {Bias }}: \lambda=0.3, \eta=0.05$ for all datasets. In $\mathrm{MF}_{\text {Side }}$ : $\lambda=0.3, \eta=0.01$ for Movielens, $\lambda=0.25, \eta=0.02$ for FilmTrust, $\lambda=0.25, \eta=0.03$ for Epinions, and $\lambda=$ $0.3, \eta=0.05$ for Lastfm. In RWR $\mathrm{Exp}_{0}: c=0.2$ for Movielens and FilmTrust dataset, and $c=0.5$ for Epinions dataset. In $\mathrm{RWR}_{\text {Imp }}: c=0.1$ for all datasets. In $\mathrm{RWR}_{\text {Bias }}: \beta=0.4, \gamma=$ $0.3, c=0.2$ for Movielens, $\beta=0.25, \gamma=0.1, c=0.2$ for FilmTrust, $\beta=0.5, \gamma=0.3, c=0.2$ for Epinions. In $\mathrm{RWR}_{\text {Side }}: \delta=2.0, c=0.2$ for Movielens, $\delta=1.0, c=0.2$ for Filmtrust, Epinions, and Lastfm.

\section{B. Performance Measures}

We employ two measures to compare performance of matrix factorization and random walk with restart. We measure global ranking (Section IV-B1) and top- $k$ prediction performance (Section IV-B2). Global ranking and top- $k$ prediction are sufficiently important measures, since recommendations provided by e-commerce services are usually ranked list of items and customers pay attention only to the top few items. Matrix factorization is known to optimize the lowest RMSE (Root Mean Square Error). However, we do not use RMSE as 
accuracy measure because RWR cannot generate predicted ratings in the same scale with the observed data.

\section{1) Ranking performance}

We compare recommendation methods with respect to how well they predict rankings of recommended items. We use Spearman's $\rho$ as the global ranking performance metric, which compares a ranked list with a ground-truth ranked list and shows correlation between the lists. $\rho$ has a value within $[-1,1]$, and a higher value of it tells the rank of the two lists are more similar.

$\rho$ is defined as average of $\rho_{u}$ for all user $u$ as stated in Equation (12). $\rho_{u}$ is defined in Equation (13). $\boldsymbol{\Omega}_{\mathbf{R}}{ }^{\text {test }}[u]$ is a set of items that $u$ gives ratings in test set, $s_{u i}$ is the rank of $i$ in a list of items sorted by predicted ratings, $s_{u i}^{*}$ is the rank of $i$ in a list of items ranked by actual ratings in test set, $\bar{s}_{u i}$ is the average of $s_{u i}$ over all items $i \in \boldsymbol{\Omega}_{\mathbf{R}}{ }^{\text {test }}[u]$, and $\bar{s}_{u i}^{*}$ is the average of $s_{u i}^{*}$ over all items $i \in \Omega_{\mathbf{R}}{ }^{t e s t}[u]$. If there are items that are tied for the same ranking, we define $s_{u i}$ and $s_{u i}^{*}$ as the average rank of all items of the same scores with $i$.

$$
\begin{gathered}
\rho=\frac{1}{|\mathbf{U}|} \sum_{u \in \mathbf{U}} \rho_{u} \\
\rho_{u}=\frac{\sum_{i \in \boldsymbol{\Omega}_{\mathbf{R}}{ }^{t e s t}[u]}\left(s_{u i}-\bar{s}_{u i}\right)\left(s_{u i}^{*}-\bar{s}_{u i}^{*}\right)}{\sqrt{\sum_{i \in \boldsymbol{\Omega}_{\mathbf{R}}{ }^{t e s t}[u]}\left(s_{u i}-\bar{s}_{u i}\right)^{2}} \sqrt{\sum_{i \in \boldsymbol{\Omega}_{\mathbf{R}}{ }^{t e s t}[u]}\left(s_{u i}^{*}-\bar{s}_{u i}^{*}\right)^{2}}}
\end{gathered}
$$

\section{2) Top- $k$ prediction performance}

We compare methods in performance on predicting top- $k$ recommended items. We measure precision@ $k$ where $k$ is the number of top items of interest. It is ratio of the number of actual positive items among the first $k$ items in recommendation list predicted by methods. The values are in range of $[0$, 1], and higher values indicate better predictive performance.

precision@ $k$ is the average of precision $@ k_{u}$ for all users $u$ as defined in Equation (14). For a user $u$, precision $@ k_{u}$ is defined in Equation (15). Actual $_{u}(k)$ is a set of top- $k$ items sorted by observed ratings given by $u$ in test set, and Predicted $_{u}(k)$ is that of top- $k$ items in test set predicted by a recommendation method.

$$
\begin{array}{r}
\text { precision } @ k=\frac{1}{|\mathbf{U}|} \sum_{u \in \mathbf{U}} \text { precision@ } k_{u} \\
\text { precision } @ k_{u}=\frac{\left|\operatorname{Actual}_{u}(k) \cap \operatorname{Predicted}_{u}(k)\right|}{k}
\end{array}
$$

\section{Performance of Methods with Explicit Feedback Data}

We compare performance of matrix factorization and random walk with restart when explicit feedback ratings are given. We find out that $\mathrm{MF}_{\text {Exp }}$ performs better than $\mathrm{RWR}_{\operatorname{Exp}}$ in this situation. Table $\mathrm{V}$ presents the results of the experiment. In using explicit feedback ratings, $\mathrm{MF}_{\text {Exp }}$ performs better than $\mathrm{RWR}_{\text {Exp }}$ in global ranking and top- $k$ prediction measures. $\mathrm{MF}_{\mathrm{Exp}}$ shows higher Spearman's $\rho$ for global ranking performance and also higher precision@1,2,3 measures for top-1, 2, 3 prediction measures for all explicit rating datasets.

\section{Performance of Methods with Implicit Feedback Data}

We compare matrix factorization and random walk with restart in their global ranking and top- $k$ prediction performance when implicit feedback ratings are given. We find out that $\mathrm{RWR}_{\text {Imp }}$ performs better than $\mathrm{MF}_{\mathrm{Imp}}$ in using implicit rating data. Table VI tells the results of this experiment. RWR $_{\text {Imp }}$ shows higher Spearman's $\rho$ and precision@1,2,3 measures for all implicit rating datasets, than $\mathrm{MF}_{\text {Imp }}$ does.

\section{E. Performance of Methods with Global Bias Terms}

With regard to global bias terms, we present performance measures of matrix factorization and random walk with restart according to the following interests: (1) whether the bias terms enhance performance, and (2) which method is better between $\mathrm{MF}_{\mathrm{Bias}}$ and $\mathrm{RWR}_{\mathrm{Bias}}$. We observe that (1) the bias terms improve recommendation performance, and (2) $\mathrm{MF}_{\text {Bias }}$ performs better than $\mathrm{RWR}_{\mathrm{Bias}}$. The experiments are conducted with only explicit feedback rating because it is inappropriate to apply global bias terms in implicit feedback ratings. The reason is that bias terms and vectors of entities are optimized to be invalid value or even 0 in $\mathrm{MF}_{\mathrm{Bias}}$ with implicit ratings. This is because the global average rating $\mu$ always offsets the implicit rating values, and thus there are no observed values left for the biases and vectors to learn from. $\mathrm{MF}_{\mathrm{Bias}}$ approximates $\mu+b_{u}+b_{i}+\mathbf{x}_{u}^{T} \mathbf{y}_{i}$ to a binarized implicit rating $p_{u i}$; however, $\mu$ and $p_{u i}$ are 1 , causing $b_{u}+b_{i}+\mathbf{x}_{u}^{T} \mathbf{y}_{i}$ to be 0 . In this case, there are no valid observed values.

Bias terms in matrix factorization are known to improve accuracy performance, decreasing RMSE [6]. We observe that bias terms also enhance global ranking and top- $k$ prediction performance in our experiments. Tables VII and VIII present the results of our experiments. Bias terms enhance the performance in both matrix factorization and random walk with restart, as the methods with bias terms show higher Spearman's $\rho$ and precision@1,2,3 compared to the ones without biases.

Our experiment results also show that $\mathrm{MF}_{\text {Bias }}$ performs better than $\mathrm{RWR}_{\mathrm{Bias}}$, which shows similar tendencies in experiment with explicit rating data in Section IV-C

\section{F. Performance of Methods with Side Information}

We focus on three aspects of matrix factorization and random walk with restart in using side information: (1) whether additional information improves recommendation performance, (2) which method performs better between $\mathrm{MF}_{\text {Side }}$ and $\mathrm{RWR}_{\text {Side }}$ with side information, and (3) which method solves the cold start problem better. In our experiments, the results are observed as follows: (1) additional information improves or shows modest decrease in the performance with explicit rating data, while the performance decreases with implicit rating data, (2) $\mathrm{MF}_{\text {Side }}$ performs better with explicit rating data and $\mathrm{RWR}_{\text {Side }}$ performs better with implicit ones, and (3) $\mathrm{MF}_{\text {Side }}$ solves the cold start problem better with explicit rating data and $\mathrm{RWR}_{\text {Side }}$ is better with implicit ones.

The ranking and top- $k$ prediction performance are improved with auxiliary information and explicit feedback ratings in general. Table IX shows the results of our experiments that compare $\mathrm{MF}_{\text {Exp }}$ and $\mathrm{MF}_{\text {Side }}$. Table $\mathrm{X}$ shows the performance 
Table V: Comparison of matrix factorization and random walk with restart in using explicit feedback datasets. MF $\mathrm{Exp}_{\text {shows }}$ better performance than $\mathrm{RWR}_{\mathrm{Exp}}$ in global ranking and top-1, 2, 3 prediction measure, as $\mathrm{MF}_{\text {Exp }}$ presents higher Spearman's $\rho$ and precision $@ 1,2,3$ than $\mathrm{RWR}_{\mathrm{Exp}}$ does.

\begin{tabular}{|c|c|c|c|c|c|c|c|c|c|c|c|c|c|}
\hline & \multirow{2}{*}{$\begin{array}{c}\text { Performance } \\
\text { summary }\end{array}$} & \multicolumn{3}{|c|}{ Spearman's $\rho$} & \multicolumn{3}{|c|}{ precision@1 } & \multicolumn{3}{|c|}{ precision@2 } & \multicolumn{3}{|c|}{ precision@3 } \\
\hline & & Movielens & FilmTrust & Epinions & Movielens & FilmTrust & Epinions & Movielens & FilmTrust & Epinions & Movielens & FilmTrust & Epinions \\
\hline & & 0.328 & 0.377 & 0.762 & 0.144 & 0.381 & & & 0.5 & & 0.365 & 0.573 & 0.6 \\
\hline RWR $_{\text {Exp }}$ & Lower & 0.238 & 0.359 & 0.616 & 0.103 & 0.319 & 0.498 & 0.197 & 0.470 & 0.585 & 0.306 & 0.549 & 0.587 \\
\hline
\end{tabular}

Table VI: Comparison of matrix factorization and random walk with restart in using implicit feedback datasets. RWR $_{\text {Imp }}$ performs better than $\mathrm{MF}_{\mathrm{Imp}}$ in global ranking and top-1,2,3 prediction, as $\mathrm{RWR}_{\mathrm{Imp}}$ presents higher Spearman's $\rho$ and precision@1,2,3 than $\mathrm{MF}_{\mathrm{Imp}}$ does.

\begin{tabular}{|c|c|c|c|c|c|c|c|c|c|}
\hline & \multirow{2}{*}{$\begin{array}{l}\text { Performance } \\
\text { summary }\end{array}$} & \multicolumn{2}{|c|}{ Spearman's $\rho$} & \multicolumn{2}{|c|}{ precision@1 } & \multicolumn{2}{|c|}{ precision@2 } & \multicolumn{2}{|c|}{ precision@3 } \\
\hline & & Lastfm & Audioscrobbler & Lastfm & Audioscrobbler & Lastfm & Audioscrobbler & Lastfm & Audioscrobbler \\
\hline $\mathrm{MF}_{\mathrm{Imp}}$ & Lower & 0.428 & 0.115 & 0.131 & 0.106 & 0.277 & 0.169 & 0.417 & 0.216 \\
\hline $\mathrm{RWR}_{\mathrm{Imp}}$ & Higher & 0.558 & 0.370 & 0.272 & 0.149 & 0.402 & 0.220 & 0.517 & 0.272 \\
\hline
\end{tabular}

Table VII: Comparison of $\mathrm{MF}_{\mathrm{Exp}}$ and $\mathrm{MF}_{\mathrm{Bias}}$ to show the effects of global bias terms in matrix factorization. Global bias terms enhance ranking and top- $k$ prediction performance. $\mathrm{MF}_{\mathrm{Bias}}$ shows better performance than $\mathrm{MF}_{\mathrm{Exp}}$ in global ranking and top-1, 2, 3 prediction measure, as $\mathrm{MF}_{\text {Bias }}$ presents higher Spearman's $\rho$ and precision $@ 1,2,3$ than $\mathrm{MF}_{\mathrm{Exp}} \mathrm{does}_{\text {. }}$

\begin{tabular}{|c|c|c|c|c|c|c|c|c|c|c|c|c|c|}
\hline & \multirow{2}{*}{$\begin{array}{l}\text { Performance } \\
\text { summary }\end{array}$} & \multicolumn{3}{|c|}{ Spearman's $\rho$} & \multicolumn{3}{|c|}{ precision@1 } & \multicolumn{3}{|c|}{ precision@2 } & \multicolumn{3}{|c|}{ precision@3 } \\
\hline & & Movielens & FilmTrust & Epinions & Movielens & FilmTrust & Epinions & Movielens & FilmTrus & Epinions & Movielens & FilmTrust & Epinions \\
\hline & & & 0377 & 0762 & & 0281 & 0507 & 0260 & 0.511 & 0653 & 0.365 & 0.573 & 0.624 \\
\hline & Higher & 0.351 & 0.424 & 0.762 & 0.152 & 0.391 & 0.624 & 0.275 & 0.551 & 0.665 & 0.375 & 0.645 & 0.631 \\
\hline Improv & 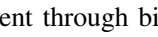 & $7.0 \%$ & $12.5 \%$ & $0 \%$ & $6.6 \%$ & $2.6 \%$ & $4.5 \%$ & $5.8 \%$ & $7.2 \%$ & $1.8 \%$ & $2.7 \%$ & $12.6 \%$ & $1.1 \%$ \\
\hline
\end{tabular}

Table VIII: Comparison of RWR $\mathrm{Exp}_{\text {and }}$ RWR $\mathrm{Bias}_{\text {ias }}$ to show the effects of global bias terms in random walk with restart. The bias terms enhance ranking and top- $k$ prediction performance. $\mathrm{RWR}_{\text {Bias }}$ performs better than RWR $\mathrm{Exp}_{\text {in }}$ global ranking and top-1,2,3 prediction measure, as $\mathrm{RWR}_{\text {Bias }}$ presents higher or equal Spearman's $\rho$ and precision $@ 1,2,3$ than $\mathrm{RWR}_{\mathrm{Exp}}$ does.

\begin{tabular}{|c|c|c|c|c|c|c|c|c|c|c|c|c|c|}
\hline & \multirow{2}{*}{$\begin{array}{l}\text { Performance } \\
\text { summary }\end{array}$} & \multicolumn{3}{|c|}{ Spearman's $\rho$} & \multicolumn{3}{|c|}{ precision@1 } & \multicolumn{3}{|c|}{ precision@2 } & \multicolumn{3}{|c|}{ precision@3 } \\
\hline & & Movielens & FilmTrust & Epinions & Movielens & FilmTrust & Epinions & Movielens & FilmTrust & Epinions & Movielens & FilmTrust & Epinions \\
\hline $\mathrm{RWR}_{\operatorname{Exp}}$ & Lower & 0.238 & 0.359 & 0.616 & 0.103 & 0.319 & 0.498 & 0.197 & 0.470 & 0.585 & 0.306 & 0.549 & 0.587 \\
\hline $\mathrm{RWR}_{\text {Bias }}$ & Higher & 0.241 & 0.369 & 0.625 & 0.105 & 0.350 & 0.498 & 0.199 & 0.487 & 0.585 & 0.312 & 0.552 & 0.589 \\
\hline Improven & nt through bias & $1.2 \%$ & $2.8 \%$ & $1.5 \%$ & $1.9 \%$ & $9.7 \%$ & $0 \%$ & $1.0 \%$ & $3.6 \%$ & $0 \%$ & $2.0 \%$ & $0.5 \%$ & $0.3 \%$ \\
\hline
\end{tabular}

comparison of $\mathrm{RWR}_{\mathrm{Exp}}$ and $\mathrm{RWR}_{\text {Side. These results present }}$ that methods using side information such as $\mathrm{MF}_{\text {Side }}$ and $\mathrm{RWR}_{\text {Side }}$ perform better in general than methods not using additional information such as $\mathrm{MF}_{\text {Exp }}$ and $\mathrm{RWR}_{\text {Exp }}$. However, the performance is worse with side information when implicit rating data are given. Table $\mathrm{XI}$ shows that $\mathrm{MF}_{\text {Side }}$ performs

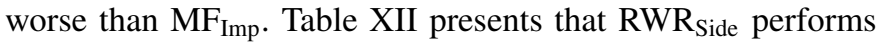
worse than $\mathrm{RWR}_{\text {Imp. These results indicate that additional }}$ information needs to be carefully handled for better accuracy.

We observe that $\mathrm{MF}_{\text {Side }}$ is better with explicit ratings and $\mathrm{RWR}_{\text {Side }}$ performs better with implicit ones. The result shows the similar tendencies in experiments with explicit or implicit data without side information in Sections IV-C and IV-D

We compare matrix factorization and random walk with restart in their ability to address the cold start problem, especially to recommend items to new users. We observe that matrix factorization performs better in solving the cold start problem when explicit ratings are given, while random walk with restart is better with implicit feedback data. We take the following steps for this experiment. First, we sample a set of cold start users $\mathbf{U}_{\text {cold }}$. We randomly sample $20 \%$ of total users for the cold users, where the chosen ones have rated more than one item and have side information. Second, we split training and test set. The training set contains side information of all users and observed ratings of users who do not belong to $\mathbf{U}_{\text {cold }}$. The test set includes observed ratings of users $\in \mathbf{U}_{\text {cold }}$. Lastly, we measure Spearman's $\rho$ for global ranking performance and precision $@ k$ for top- $k$ prediction performance. Tables XIII and XIV show the result that $\mathrm{MF}_{\text {Side }}$ better solves the cold start problem with explicit feedback data and $\mathrm{RWR}_{\text {Side }}$ is better with implicit ratings.

\section{G. Discussion}

What are the reasons for good or bad performance of MF and RWR in various settings of recommendations? We observe interesting tendencies over various performance measures in our experiments:

- Matrix factorization performs better with explicit rating datasets, while random walk with restart performs better with implicit ones.

- Global bias terms improve recommendation performance.

- Additional information enhance performance of recommendations with explicit rating data, while it makes worse performance with implicit rating data.

Why matrix factorization conforms well to explicit ratings while random walk with restart applies well to implicit ones? Random walk with restart is influenced by proximity of nodes or the number of hops, while explicit rating scores occasionally contradict the concept of the proximity. For example, random walk with restart gives a higher RWR score on a 1-hop 
Table IX: Comparison of $\mathrm{MF}_{\text {Exp }}$ and $\mathrm{MF}_{\text {Side }}$ to show whether side information improves the recommendation performance in matrix factorization with restart when explicit feedback data are given. The global ranking performance is enhanced with side information and top- $k$ prediction performance also improves except in several cases, as MF $_{\text {Side }}$ presents higher Spearman's $\rho$ in all cases and higher precision $@ 1,2,3$ in almost all cases than $\mathrm{MF}_{\mathrm{Exp}}$ does.

\begin{tabular}{|c|c|c|c|c|c|c|c|c|c|c|c|c|c|}
\hline & $\begin{array}{l}\text { Performance } \\
\text { summary }\end{array}$ & $\begin{array}{r}\mathrm{S} \\
\text { Movielen }\end{array}$ & pearman's & $\rho$ & $\mid \begin{array}{r}p \\
\text { Movielens }\end{array}$ & $\frac{\text { ecision@ }}{\text { FilmTrust }}$ & $\frac{1}{\text { Epinions }}$ & $\begin{aligned} p r \\
\text { Movielens }\end{aligned}$ & $\frac{\text { recision@ }}{\text { FilmTrust }}$ & $\begin{array}{l}2 \\
\text { Epinions }\end{array}$ & $\mid \begin{array}{r}p \\
\text { Movielen }\end{array}$ & $\frac{\text { recision }}{\text { FilmTrus }}$ & $\frac{3}{\text { Epinions }}$ \\
\hline $\mathrm{MF}_{\mathrm{Exp}}$ & Lower & 0.328 & 0.377 & 0.762 & 0.144 & 0.381 & 0.597 & 0.260 & 0.514 & 0.653 & 0.365 & 0.573 & 0.624 \\
\hline $\mathrm{MF}_{\text {Side }}$ & Higher & 0.361 & 0.416 & 0.793 & 0.147 & 0.403 & 0.650 & 0.269 & 0.524 & 0.628 & 0.375 & 0.574 & 0.577 \\
\hline \multicolumn{2}{|c|}{ Improvement through side info } & $10.0 \%$ & $10.3 \%$ & $4.0 \%$ & $2.0 \%$ & $5.8 \%$ & $8.9 \%$ & $3.5 \%$ & $1.9 \%$ & $-3.8 \%$ & $2.7 \%$ & $0.2 \%$ & $-7.6 \%$ \\
\hline
\end{tabular}

Table X: Comparison of RWR $\mathrm{Exp}_{\text {and }}$ RWR $\mathrm{R}_{\text {Side }}$ to show whether side information improves the recommendation performance in random walk with restart when explicit feedback data are given. The global ranking performance is enhanced with side

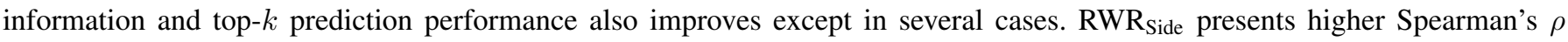
in all cases and higher precision@1,2,3 in almost all cases than $\mathrm{RWR}_{\operatorname{Exp}}$ does.

\begin{tabular}{|c|c|c|c|c|c|c|c|c|c|c|c|c|c|}
\hline & $\begin{array}{l}\text { Performance } \\
\text { summary }\end{array}$ & \begin{tabular}{|r}
$\mathrm{S}_{\mathrm{f}}$ \\
Movielens
\end{tabular} & $\begin{array}{l}\text { earman's } \\
\text { FilmTrust }\end{array}$ & $\frac{\rho}{t \text { Epinions }}$ & $\begin{array}{r}p r \\
\text { Movielens }\end{array}$ & $\frac{\text { ecision@ }}{\text { FilmTrust }}$ & $\frac{1}{\text { Epinions }}$ & $\begin{array}{r}p r \\
\text { Movielens }\end{array}$ & $\frac{\text { recision@ }}{\text { FilmTrust }}$ & $\frac{2}{\text { Epinions }}$ & \begin{tabular}{r|r}
$p r$ \\
Movielens
\end{tabular} & $\frac{\text { ecision } @}{\text { FilmTrus }}$ & $\frac{3}{\text { Epinions }}$ \\
\hline $\mathrm{RWR}_{\operatorname{Exp}}$ & Lower & 0.238 & 0.359 & 0.616 & 0.103 & 0.319 & 0.498 & 0.197 & 0.470 & 0.585 & 0.306 & 0.549 & 0.587 \\
\hline $\mathrm{RWR}_{\text {Side }}$ & Higher & 0.239 & 0.384 & 0.678 & 0.103 & 0.357 & 0.578 & 0.197 & 0.438 & 0.573 & 0.306 & 0.540 & 0.542 \\
\hline Improven & through side info & $0.4 \%$ & $7.0 \%$ & $10.0 \%$ & $0 \%$ & $11.9 \%$ & $8.9 \%$ & $0 \%$ & $-6.9 \%$ & $-2.1 \%$ & $0 \%$ & $-6.2 \%$ & $-7.7 \%$ \\
\hline
\end{tabular}

Table XI: Comparison of $\mathrm{MF}_{\mathrm{Imp}}$ and $\mathrm{MF}_{\text {Side }}$ to show whether side information improves the recommendation performance with implicit rating. The performance decreases with side information. $\mathrm{MF}_{\text {Side }}$ shows worse performance than $\mathrm{MF}_{\text {Imp }}$ in global ranking and top-1, 2, 3 prediction measure, as $\mathrm{MF}_{\text {Side }}$ presents lower Spearman's $\rho$ and precision $@ 1,2,3$ than $\mathrm{MF}_{\text {Imp }}$ does.

\begin{tabular}{c|c|c|c|c|c}
\hline & Performance & Spearman's $\rho$ & precision $@ 1 ~$ & precision $@ 2$ & precision $@ 3$ \\
\cline { 2 - 6 } & summary & Lastfm & Lastfm & Lastfm & Lastfm \\
\hline $\mathrm{MF}_{\text {Imp }}$ & Higher & $\mathbf{0 . 4 2 8}$ & $\mathbf{0 . 1 3 1}$ & $\mathbf{0 . 2 7 7}$ & 0.263 \\
$\mathrm{MF}_{\text {Side }}$ & Lower & 0.388 & 0.130 & $-5.1 \%$ & 0.397 \\
\hline \multicolumn{2}{c|}{ Improvement through side info } & $-9.4 \%$ & $-0.8 \%$ & $-4.8 \%$ \\
\hline
\end{tabular}

Table XII: Comparison of RWR $\mathrm{Imp}_{\mathrm{Im}}$ and RWR $\mathrm{Ride}_{\text {side }}$ to show whether side information improves or decrease the recommendation performance when implicit rating data are given. The performance decreases with side information. RWR $_{\text {Side }}$ shows less performance than $\mathrm{RWR}_{\mathrm{Imp}}$ in global ranking and top-1,2,3 prediction measure, as $\mathrm{RWR}_{\text {Side }}$ presents lower Spearman's $\rho$ and precision $@ 1,2,3$ than $\mathrm{RWR}_{\mathrm{Imp}}$ does.

\begin{tabular}{c|c|c|c|c|c}
\hline & Performance & Spearman's $\rho$ & precision@1 & precision@2 & precision $@ 3$ \\
\cline { 2 - 6 } & summary & Lastfm & Lastfm & Lastfm & $\mathbf{0 . 4 0 2}$ \\
\hline RWR $_{\text {Imp }}$ & Higher & $\mathbf{0 . 5 5 8}$ & $\mathbf{0 . 2 7 2}$ & 0.385 & 0.505 \\
RWR $_{\text {Side }}$ & Lower & 0.544 & -242 & $-4.3 \%$ & $-2.4 \%$ \\
\hline Improvement through side info & $-2.6 \%$ & $-1.1 \%$ & \\
\hline
\end{tabular}

negative item (e.g. an item that a user $u$ gives a low rating) than a 3-hop positive item (e.g. an item preferred by another user whose taste is similar to $u$ ). Implicit data only include users' positive behavior on items and do not include a shortcut between negatively related nodes. Therefore, random walk with restart fits well with implicit data rather than explicit ones. This explains A1, A2, A4, A6, and A7 in the question and answer list at the beginning page in Section IV. As regards $\mathrm{A} 4, \mathrm{MF}_{\text {Bias }}$ performs better than $\mathrm{RWR}_{\text {Bias }}$ because we use only explicit ratings in comparison of them as explained in Section IV-E

Global bias terms enhance the global ranking and top- $k$ prediction performance in both matrix factorization and random walk with restart. Bias factors improve the performance because the methods learn not only user-item interactions but also nodes' specific properties accounting for much of the variation in observed ratings [6]. This explains A3 in the question and answer list.

Additional information is used well with explicit feedback data, but not with implicit ones. Our conjecture is that side information is noisy, and the noise further debases the quality of information in implicit feedback ratings that are also noisy. This possibly explains A5 in the question and answer list.

\section{CONClusion}

We provide a comparative study of matrix factorization (MF) and random walk with restart (RWR) in recommender system. We suggest four tasks according to various recommendation scenarios. We suggest recommendation methods based on MF and RWR, and show that each scenario has its corresponding MF and RWR methods. Especially, we devise a new RWR method using global bias terms, and the new method improves the recommendation performance. We provide extensive experimental results that compare MF and RWR for the various tasks and explain insight for the reasons behind the results. We observe that MF and RWR behave differently according to whether input ratings are explicit or implicit. We also observe that global popularities of nodes represented as biases are useful in improving the recommendation performance. Finally, 
Table XIII: Comparison of $\mathrm{MF}_{\text {Side }}$ and $\mathrm{RWR}_{\text {Side }}$ to evaluate which method better solves the cold start problem when explicit ratings are given. $\mathrm{MF}_{\text {Side }}$ performs better in this case. $\mathrm{MF}_{\text {Side }}$ shows better performance than $\mathrm{RWR}_{\text {Side }}$ in global ranking and top-1,2,3 prediction measure, as $\mathrm{MF}_{\text {Side }}$ presents higher Spearman's $\rho$ and precision $@ 1,2,3$ than $\mathrm{RWR}_{\text {Side }}$ does.

\begin{tabular}{|c|c|c|c|c|c|c|c|c|c|c|c|c|c|}
\hline & \multirow{2}{*}{$\begin{array}{c}\text { Performance } \\
\text { summary }\end{array}$} & \multicolumn{3}{|c|}{ Spearman's $\rho$} & \multicolumn{3}{|c|}{ precision@1 } & \multicolumn{3}{|c|}{ precision@2 } & \multicolumn{3}{|c|}{ precision@3 } \\
\hline & & Movielens & FilmTrust & Epinions & Movielens & FilmTrust & Epinions & Movielens & FilmTrust & Epinions & Movielens & FilmTrust & Epinions \\
\hline & & 0.310 & 0.248 & 0.640 & & & & & 0.255 & & 0.1 & 0.288 & 0.448 \\
\hline RWR $_{\text {Side }}$ & Lower & 0.231 & 0.201 & 0.399 & 0.032 & 0.146 & 0.362 & 0.064 & 0.237 & 0.416 & 0.105 & 0.279 & 0.446 \\
\hline
\end{tabular}

Table XIV: Comparison of $\mathrm{MF}_{\text {Side }}$ and $\mathrm{RWR}_{\text {Side }}$ to evaluate which method better solves the cold start problem when implicit ratings are given. $\mathrm{RWR}_{\text {Side }}$ performs better in this case. $\mathrm{RWR}_{\text {Side }}$ shows better performance than $\mathrm{MF}_{\text {Side }}$ in global ranking and top-1,2,3 prediction measure, as $\mathrm{RWR}_{\text {Side }}$ presents higher Spearman's $\rho$ and precision $@ 1,2,3$ than $\mathrm{MF}_{\text {Side }}$ does.

\begin{tabular}{c|c|c|c|c|c}
\hline & Performance & Spearman's $\rho$ & precision $@ 1 ~$ & precision@2 & precision@3 \\
\cline { 3 - 6 } & summary & Lastfm & Lastfm & Lastfm & Lastfm \\
\hline MF $_{\text {Side }}$ & Lower & 0.207 & 0.026 & 0.041 & 0.058 \\
RWR $_{\text {Side }}$ & Higher & $\mathbf{0 . 4 4 0}$ & $\mathbf{0 . 0 7 0}$ & $\mathbf{0 . 1 0 0}$ & $\mathbf{0 . 1 4 9}$ \\
\hline
\end{tabular}

we observe that side information improves recommendation quality with explicit feedback, while degrades it with implicit feedback.

\section{ACKNOWLEDGMENT}

This work was supported by ICT R\&D program of MSIP/IITP. [2013-0-00179, Development of Core Technology for Contextaware Deep-Symbolic Hybrid Learning and Construction of Language Resources]. U Kang is the corresponding author.

\section{REFERENCES}

[1] P. Resnick and H. R. Varian, "Recommender systems," Communications of the ACM, vol. 40, no. 3, pp. 56-58, 1997.

[2] K. Shin and U. Kang, "Distributed methods for high-dimensional and large-scale tensor factorization," in ICDM, 2014.

[3] K. Shin, L. Sael, and U. Kang, "Fully scalable methods for distributed tensor factorization," IEEE Trans. Knowl. Data Eng., vol. 29, no. 1, pp. 100-113, 2017.

[4] J. B. Schafer, J. Konstan, and J. Riedl, "Recommender systems in ecommerce," in Proceedings of the 1st ACM conference on Electronic commerce. ACM, 1999, pp. 158-166.

[5] H. Chen, X. Li, and Z. Huang, "Link prediction approach to collaborative filtering," in Digital Libraries, 2005. JCDL'05. Proceedings of the 5th ACM/IEEE-CS Joint Conference on. IEEE, 2005, pp. 141-142.

[6] Y. Koren, R. Bell, and C. Volinsky, "Matrix factorization techniques for recommender systems," Computer, vol. 42, no. 8, 2009.

[7] Y. Koren, "The bellkor solution to the netflix grand prize," Netflix prize documentation, vol. 81, pp. 1-10, 2009.

[8] L. Baltrunas, B. Ludwig, and F. Ricci, "Matrix factorization techniques for context aware recommendation," in Proceedings of the fifth ACM conference on Recommender systems. ACM, 2011, pp. 301-304.

[9] Y. Song, S. Dixon, and M. Pearce, "A survey of music recommendation systems and future perspectives."

[10] J. Naruchitparames, M. H. Güneş, and S. J. Louis, "Friend recommendations in social networks using genetic algorithms and network topology," in Evolutionary Computation (CEC), 2011 IEEE Congress on. IEEE, 2011, p. 22072214.

[11] D. Liben-Nowell and J. Kleinberg, "The link-prediction problem for social networks," journal of the Association for Information Science and Technology, vol. 58, no. 7, pp. 1019-1031, 2007.

[12] C. Wang and D. M. Blei, "Collaborative topic modeling for recommending scientific articles," in Proceedings of the 17th ACM SIGKDD international conference on Knowledge discovery and data mining. ACM, 2011, p. 448456.

[13] P. Covington, J. Adams, and E. Sargin, "Deep neural networks for youtube recommendations," in Proceedings of the 10th ACM Conference on Recommender Systems. ACM, 2016, pp. 191-198.

[14] H. Li, Y. Ge, R. Hong, and H. Zhu, "Point-of-interest recommendations: Learning potential check-ins from friends."

[15] G. Linden, B. Smith, and J. York, "Amazon. com recommendations: Item-to-item collaborative filtering," IEEE Internet computing, vol. 7, no. 1 , pp. $76-80,2003$.
[16] M.-S. Shang, Y. Fu, and D.-B. Chen, "Personal recommendation using weighted bipartite graph projection," in Apperceiving Computing and Intelligence Analysis, 2008. ICACIA 2008. International Conference on. IEEE, 2008, pp. 198-202.

[17] X. Su and T. M. Khoshgoftaar, "A survey of collaborative filtering techniques," Advances in artificial intelligence, vol. 2009, p. 4, 2009.

[18] I. Barjasteh, R. Forsati, F. Masrour, A.-H. Esfahanian, and H. Radha, "Cold-start item and user recommendation with decoupled completion and transduction," in Proceedings of the 9th ACM Conference on Recommender Systems, ser. RecSys '15. New York, NY, USA: ACM, 2015, pp. 91-98.

[19] K. Zhou, S.-H. Yang, and H. Zha, "Functional matrix factorizations for cold-start recommendation," in Proceedings of the 34th international ACM SIGIR conference on Research and development in Information Retrieval. ACM, 2011, pp. 315-324.

[20] H. Tong, C. Faloutsos, and J.-y. Pan, "Fast random walk with restart and its applications," in Data Mining, 2006. ICDM'06. Sixth International Conference on. IEEE, 2006, pp. 613-622.

[21] U. Kang, H. Tong, and J. Sun, "Fast random walk graph kernel," 2012.

[22] U. Kang, M. Bilenko, D. Zhou, and C. Faloutsos, "Axiomatic analysis of co-occurrence similarity functions," CMU-CS-12-102, 2012.

[23] K. Shin, J. Jung, L. Sael, and U. Kang, "Bear: Block elimination approach for random walk with restart on large graphs," in SIGMOD, 2015.

[24] J. Jung, K. Shin, L. Sael, and U. Kang, "Random walk with restart on large graphs using block elimination," ACM Trans. Database Syst., vol. 41, no. 2, p. 12, 2016.

[25] J. Jung, N. Park, L. Sael, and U. Kang, "Bepi: Fast and memory-efficient method for billion-scale random walk with restart," in Proceedings of the 2017 ACM International Conference on Management of Data, SIGMOD Conference 2017, Chicago, IL, USA, May 14-19, 2017, 2017, pp. 789804.

[26] I. Konstas, V. Stathopoulos, and J. M. Jose, "On social networks and collaborative recommendation," in Proceedings of the 32nd international ACM SIGIR conference on Research and development in information retrieval. ACM, 2009, pp. 195-202.

[27] J. Jung, W. Jin, L. Sael, and U. Kang, "Personalized ranking in signed networks using signed random walk with restart," in IEEE 16th International Conference on Data Mining, ICDM 2016, December 1215, 2016, Barcelona, Spain, 2016, pp. 973-978.

[28] H. Yildirim and M. S. Krishnamoorthy, "A random walk method for alleviating the sparsity problem in collaborative filtering," in Proceedings of the 2008 ACM Conference on Recommender Systems, ser. RecSys '08. New York, NY, USA: ACM, 2008, pp. 131-138.

[29] Y. Hu, Y. Koren, and C. Volinsky, "Collaborative filtering for implicit feedback datasets," in Data Mining, 2008. ICDM'08. Eighth IEEE International Conference on. Ieee, 2008, pp. 263-272.

[30] A. N. Langville and C. D. Meyer, Google's PageRank and beyond: The science of search engine rankings. Princeton University Press, 2011.

[31] G. Guo, J. Zhang, and N. Yorke-Smith, "A novel bayesian similarity measure for recommender systems." 